at two hospitals in Greece with hirsutism and/ or menstrual abnormalities and/or subfertility. In 634 women the diagnosis of PCOS was established; a control group of 108 women with no evidence of PCOS was selected. Patients (women diagnosed with PCOS) were subdivided into three groups according to whether they had biochemical or clinical hyperandrogenism, or no evidence of hyperandrogenism. Patients with hyperandrogenism were further subdivided according to the presence or absence of polycystic ovaries on ultrasound and of chronic anovulation. Participants' hormone levels, their BMI and insulin resistance index were determined.

The 'classic' phenotype of chronic anovulation with hyperandrogenism was present in $86 \%$ of patients. Overall, biochemical hyperandrogenism was present in $81 \%$ and clinical hyperandrogenism in $13 \%$ of patients. Insulin resistance was greater in anovulatory patients with biochemical hyperandrogenism than in controls.

The study found a positive association of follicle numbers with androstenedione levels, and a negative association with insulin sensitivity. The odds ratio of women with PCOS having more than 12 follicles or having an ovarian volume $>10 \mathrm{~cm}^{3}$ on ultrasonography was significantly greater compared with controls.

The authors conclude that the more insulinresistant phenotypes were also the more hyperandrogenic and the most prevalent phenotypes. Long-term follow-up studies might confirm the clinical usefulness of the PCOS subclassification.

Original article Diamanti-Kandarakis E and Panidis D (2007) Unravelling the phenotypic map of polycystic ovary syndrome (PCOS): a prospective study of 634 women with PCOS. Clin Endocrinol (Oxf) 67: 735-742

\section{Causes of pregnancy loss differ between women with type 1 and those with type 2 diabetes}

Cundy et al. recently published data on the causes of pregnancy loss in women with type 1 or type 2 diabetes. Data were collected prospectively over a 20-year period at a diabetes pregnancy service in Auckland, New Zealand. The causes of pregnancy loss were assigned to one of five major categories (major congenital abnormalities, prematurity, chorioamnionitis, unexplained stillbirth, asphyxia during delivery) or to other causes.

There were 1,200 pregnancies in 903 women with diabetes. In 325 of these, diabetes was diagnosed during pregnancy and confirmed with a glucose tolerance test 6 weeks postpartum; 314 of the 325 women had type 2 diabetes. Mean $\mathrm{HbA}_{1 \mathrm{c}}$ values were similar in women with known type 1 and type 2 diabetes. Women with type 2 diabetes had a significantly greater BMI and were more commonly of nonEuropean descent than women with type 1 diabetes. A total of 42 pregnancy losses were recorded. More than $75 \%$ of pregnancy losses in women with type 1 diabetes were caused by congenital anomalies or prematurity. In women with type 2 diabetes, more than $75 \%$ of losses were caused by stillbirth, chorioamnionitis or birth asphyxia. Stillbirth was significantly more prevalent in women with type 2 diabetes than in those with type 1 diabetes.

The authors conclude that, although the rate of pregnancy loss was similar between women with type 1 and type 2 diabetes, the causes differed. They suggest that additional risk factors such as obesity have a substantial impact.

Original article Cundy T et al. (2007) Differing causes of pregnancy loss in type 1 and type 2 diabetes. Diabetes Care 30: $2603-2607$

\section{OGTT should be performed when patients with acute myocardial infarction are discharged}

The prevalence of previously undetected glucose abnormalities is high among patients with acute myocardial infarction. The WHO currently recommends that a standardized oral glucose tolerance test (OGTT) should be performed to classify glucose abnormalities. Wallander et al. have evaluated the long-term reliability of early classification of glucose abnormalities using an OGTT in patients with acute myocardial infarction without previously diagnosed type 2 diabetes. The analysis is based on data from 122 patients from the GAMI (Glucose tolerance in Acute Myocardial Infarction) study, in whom a standardized OGTT was performed when they were discharged from hospital and after 3 and 12 months.

Out of those patients newly diagnosed with type 2 diabetes after an OGTT at discharge, after 12 months $64 \%$ were still classified as 